A veriy carefial examination of the heart now faifed to detect the slightest trace of an anæmic murmur. This favourable condition continued until the fourth day, when he had a hrmorrhage from the kidney (the second one). His corpuscles were again estimated; and found to be increased from 16 per cent. to 44 per cent. He died the same night:

Neoropoy, twelve hours after death.-We ate indebted to Dr. Young for his kindness in making a careful and exhaustive post mortem examination. His report was as follows. The body was well-developed, but considerably emaciated; everywhere the integument was jaundiced; the conjunctivæ were also faintly bile-stained. An anæmic appearance was universal. Internal examination showed generally an anæmic condition of the organs, most of which presented a paler but yellower colour than normal; whilst they were also of softer consistency. The muscles were pale and flabby ; this was most marked in the walls of the heart, which, beyond dilatation of its cavities, appeared in other respects fairly normal. Its various cavities (but especially the right) contained dark fluid blood, which showed no tendency to coagulation, whilst it possessed a faint acid reaction. Each lung showed slight hypostatic congestion, and was somewhat cedematous, the fluid being yellowish in colour. The smaller bronchial tubes. were surrounded by a slightly increased amount of peribronchitic tissue. The pericardiac and pleural sacs contained a little clear yellowish fluid, but otherwise were normal. The mucous membrane of the small intestines was thickened and granular. The liver was enlarged and fatty; the kidneys slightly granular. All the other organs were healthy. Sections of the sternum and ribs showed the cancellous tissue filled with deep red coloured marrow, which in appearance closely resembled splenic pulp. Microscopic examination showed the characters of embryonic medulla, nucleated red blood-corpuscles, large granular cells, leucocytes of various forms, and giant-cells. Microscopic examination of the different organs revealed only the fatty degenerative changes indicated by the naked-eye appearances. In the case of the kidneys; there was also some increase of interstitial connective tissue.

REMARKs.-We should like to call your attention to the following points in this case, which we think are specially deserving of notice. First, the extreme retinal extravasation was suggestive of advanced pernicious anæmia. Faint hæmorrhages do occur, but very rarely, in chronic anæmia. Of fifty cases examined by Dr. Saundby and Mr. Eales of Birmingham, only one showed a small hæmorrbage; five showed some neuro-retinitic changes; the remainder were free from any retinal lesions. Dr. Gowers, who has examined cases of chronic anæmia with the same object, observes that the pallor of the fundus may be very marked in chlorosis; but, where the corpuscular richness has been reduced even so low as 26 per cent., hæmorrhages are extremely rare. This observation is of special interest when contrasted with the cases of pernicious anæmia mentioned later, where, with less destruction of corpúscles, hæmorrhages did sppear.

With tolerable certainty, retinal hæmorrhages occur in.purpura hæmorrhagica, at least when very advanced. In leukhæmia, the tendency to hæmorrhage of the retina is extremely strong; bat the effisions of blood are in this disease equatorial, and issociated with small leukbæmic tumours, which appear as spots in the choroid.

The tendency to hæmorrhage may, indeea, be considered as even stronger in leukhæmia than in pernicious anæmia. Dr. Gowers reports cases where they have occurred with the blood containing 50 per cent. of red corpuscles. But, in the advanced stages of pernicious anæmia, such hæmorrhages as are shown in the drawing probably always occur." At least, they have always been found when sought for. The observations of Hormer and Quincke on fortysix cases of the kind may well assure us that these hæmorrhages are constant in their appearance.

In a paper by Dr. Stephen Mackenzie (vol. i, ophth. Trans.) will also be 'found' a record of two cases, which show clearly that, at that period of this disease, hæmorrhages occur. The following is a brief abstract. His first patient, a youth, aged 18, had been under daily observation for three weeks before the hæmorrhages appeared; his corpuscular richness on admission being 51 per cent.; and the interest centres in the fact that it was not until the percentage dropped to 32 that the hæmorrhages appeared. They increased with each decrease of the percentage till, at 13 per cent., the patient died. Daily charts of the hamorrhagic infiltration accompany his paper.

In the same paper is another equally suggestive case, in a man aged 59. The corpuscular richness was estimated two days after the first hæmorrhage; it was then 27 per cént. The two days wbuld probably have made the difference between it and" the'ptes vious case, so that, taking these two of widely differing' ages sias fairly representative, we may asstame that retinal hemotrhages oorit: mence when the corpuscles have dropped to 32 per cent., or there abouts.

The next point to be noted is, the inability of the patient to tole:rate healthy blood. This was shown by his alarming condition after the transfusion, and the apparent relief given by the hæmorrhage from the bowel. But we may fairly place against these circumstances the complete removal of the anxmic murmurs, and the increase of corpuscular richness of the blood after the operation.

It may be a question whether the case was not too far advanced to admit of cure by any method of treatment. Quinquaud remarks that those cases are invariably fatal in which the amount of hæmoglobin is diminished below one-fifth the normal; and in this case it was only one-sixth.

According to Dr. Coupland (Gulstonian Lectures on Anæmia 1881), the operation of transfusion was performed in twenty out of the 110 cases to which he refers. And the six recoveries, one of which was not permanent, all occurred in Quincke's practice. Dr. Coupland thinks that " his success in these cases, may be attributed to his having resorted to transfusion at a less advanced stage of the disease than is usually the case." His first cases were invariably fatal, and in his commentary upon these, he pointed out "The inutility of deferring transfusion until the heart is already advanced in degeneration, and restoration is well-nigh hopeless."

We are quite unable to explain the remarkable increase of the corpuscles from 16 to 44 per cent. after transfusion. We, therefore, have recorded the fact without attempting to explain it. Something of the same kind has also been noted before. Thus, Dr. Broadbent (in Tanner's Practice of Mredicine, seventh edition, p. 18) mentions a case of anamia in which transfusion was employed four times in a fortnight, eight ounces of healthy blood being injected each time. "At first the man seemed to rally, but at a later period he was ob. served to exhibit symptoms of pulmonary and cerebral congestion. He died a few hours after the fourth operation. No morbid alterations of any importance were found on making the post mortem examination, only the organs were pale, flabby, anæmic. It was remarked that the blood, which at first was quite watery and poor, had grown much thicker under the process of transfusion." The writer remarks: "The question naturally arises whether the injection of healthy blood can sufficiently excite the blood-forming organs to such a normal action, as to keep ap the supply of nutritive material."

The case is also interesting in one or two of its negative aspects. Thus its origin could not be attributed to poor diet, to malaria, to hardship, to hæmorrhage, or to nervous shock. There was no marked diminution in the size of the aorta, such as sometimes occurs in chlorosis ; nor any notable sign of an increase of iron in the liver or other organs, unless the general yellow colour of these viscera could be ascribed to this cause.

With regard to the question of the distinction of so-called per nicious anæmia from chlorosis on the one hand, and from ordinary chronic anæmia on the other, we are unable to offer any decisive opinion. The only points of distinction from these complaints in the case before us seem to have been its progressive character, and the occurrence and nature of the retinal hæmorrhages. We, therefore, simply record the case, in the hope that at some future time it may assist in determining the question.

\section{PALATABLE DRUGS FOR CHILDREN.}

BY FREDERICK CHURCHILL, M.D., F.R.C.S., Surgeon to, and Member of the Drug Committee, Victoria Hospital for Children.

WE owe it, probably, much to the persistency with which practitioners of the sterner sort have impressed their rhubarb and black draughts upon rebellious children, in defiance of the protestations of nurses and mothers, that "the tasteless globule" has found such favour with the weaker sex. I could tell of several cases where the children have been entrusted to the care of a homcopath, while the parents luxuriate under the usual heroic treatment of the orthodox practitioner. We must not forget to swim with the tide. Children of this enlightened age are far more pampered and spoilt than those of the previous generation. Mothers seem 'unable to control their feelings; or, it may be, that, with a smattering of physic-lore, they find that there is no longer any necessity to cling to the once inevitable and nauseous potion. We must say a word, too, for the children: None of us like compulsion. It must not be forgotten that there is 
often more harm done to a child's nervous system, by cramming the draught down its throat, than all the good the nauseous drug was supposed to effect. Children will often take days to recover their equilibrium after a protracted encounter with the medicine-glass in the nursery, under the stern discipline of a would-be conscientious nurse. Judging from the varied susceptibilities and dispositions of the children under $\mathrm{my}^{\prime}$ care, some of them having very resolute wills, others possessing - I cannot say endowed with-mothers of a pronounced æesthetic temperament, to whom everything is a bore, except a novel to read and a sofa to lie upon, it becomes most important to formulate a line of treatment that will satisfy such requirements.

This class of children are generally ruled by a domineering old woman they call "nurse", displaying a maximum of "tall talk," with a minimum of what she delights to call "common sense" (and very common, indeed, it proves to be). The medical man must cultivate a habit of attacking such a stronghold of prejudice and conceit by a series of carefully planned flank movements, in such a way that the [nursery magnate may be drawn, against her own convictions, into a pliable frame of mind, sufficient to enable the medical man's physic and regimen to stand a chance of being attended to.

To attempt to invade the sanctum of a nursery where the lady. paramount is cajoled into the idea that "nurse is a treasure", and prefers rather to foster the notion, than to care to have her eyes opened to the actual state of reigning ignorance, requires all the practical art of the medical man gradually to overcome and remedy.

Undoubtedly the ailments under which children for the most part suffer, belong to the preventable class. They are due sometimes to overfeeding; very often to neglect, especially of the calls of nature; and very much to general bad management. With this view, it may be well to presume that the best and most approved mode of treatment for habitual torpidity of the bowels is not medicine, but an enema of soap and water, with occasionally a little castor or olive oil added to the injection. If this do not succeed, and the child's appetite begins to fail, it is an indication for administering medicine by the mouth.

Fortunately, the art of the apothecary comes in to our aid, and we are now enabled to give the most nauseous of drugs-castor-oilabsolutely free from taste and smell, while it retains the full aperient properties of ordinary castor-oil. Messrs. Allen and Hanburys themselves advise that it should be shaken up with three or four times its bulk of hot milk. The viscidity of the oil is thus avoided, and the emulsion produced is scarcely distinguishable from warm rich milk.

If it be desirable to administer an aperient that will act more directly on the liver, and to avoid the unpleasant effects which often arise after taking "oil," the compound rhubarb pill will be found a serviceable aperient. Of course, some new method for its administration will be desired, which I shall now detail. Either an ordinary five-grain pill may be cut up, and a portion of it broken in small pieces may be buried in a chocolate-cream, which the youngest child will take with avidity; or for children of, say five years and upwards, I have given one-half and one-fourth of a grain of this pill, thinly coated. Half-a-dozen or so may be taken, like "hundreds and thousands," and washed down with milk or water.

The medicated fruit lozenges are very useful, o.g., tamar indien and laxora lozenges. Podophyllin is probably one of the active ingredients in these lozenges. Only a small portion of a lozenge must be given to a child. The objection found with these is that they sometimes "gripe" the little patient. Next to these, perhaps, in efficiency and palatability is the compound liquorice powder, containing senna powder. About a teaspoonful stirred up with warm milk may be taken at bedtime, and a little chloric ether added (about ten to twenty drops). Very few children will object to take fluid magnesia or the calcined magnesia, especially if flavoured with the syrup of mulberry or orange.

I have succeeded in masking the taste of many powders by the addition of powdered "rose" lozenges. I very seldom prescribe Gregory's powder, on account of its nauseous character and bulk. I prefer to combine the rhubarb with bicarbonate of soda about five grains of each. This makes a much more miscible and manageable powder. Given in jam, honey or golden syrup, the taste is altogether covered.

Children will sometimes take the "baume de vie," or decoction of aloes, without objecting much. A little of this rubbed into the stomach of infants will suffice sometimes to procure an action of the bowels. The extract of liquorice may be added to the decoction until the bitter taste is sufficiently masked. Children have not really such an aversion to it, for I have known them to lick off the aloes from their fingers when put on to prevent them from sucking them.
Powdered aloes, about half a teaspoonful, may be given mixed with brown sugar. The electuary of senna is taken without difficulty by some children, also the syrup of senna and the infusion with prunes. The effervescing purgative lemonade is a very agreeable drink, as also half a seidlitz-powder flavoured with lemon-juice.

Turning now to febrifuge mixtures, there is not much need of flavouring to mask the flavour of these. Sweet nitre, acetate of ammonia, spirits of chloroform, are all pleasant drugs to take. The nitrate and chlorate of potash are rather saltish, but the sal prunelle and Wyeth's compressed tablets will be taken by the bigger children without much protest. The syrups of orange, lemon, and mulberry, will come in as agreeable and cooling adjuncts. Cough-mixtures can generally be made very pleasant by the addition of syrup of squills of tolu, etc.

As regards tonics, some considerable skill will be necessary efficiently to cover the bitter flavour. Children will take the saccharated carbonate of iron very well, and also steel wine; but if we attempt to give the bitter infusions, there is sure to be rebellion in the nursery. Quinine-one of the most valuable medicines for children -can be given without difficulty, either in the form of pill or, which I prefer, dissolved in syrup of orange, without the addition of any water. This effectually covers the flavour. Quinine wine is useful for the elder children.

Chemical food is, of course, taken with relish, and if recently made is a serviceable tonic; but the phosphates, from their insolubility, throw down very much. The compound solution of the hypophosphites, in ten-minim doses, and the hypophosphite wine, forms a perfect substitute for Parrish's food. Besides having iron in a form which is easily absorbed, the hypophosphite of magnesia serves as an useful antacid and stomachic in this combination.

With a view of putting into practical form these few suggestions, and to systematise the irregular but constant attempts of mothers to keep a little dispensary of their own, I have instructed Messrs. Savory and Moore to fit up a nursery medicine-chest, with a companion guide, to assist mothers, especially those residing in the colonies and far away from medical aid, to treat their own children in such emergencies.

\section{EXCISION OF AN ENORMOUS TUMOUR (PROBABLY OSTEO-FIBROMA) GROWING FROM THE BASE OF} THE SKULL, PRESSING ON AND PARTIALLY ABSORBING THE RIGHT SUPERIOR MAXILLA, AND NECESSITATING THE REMOVAL OF THAT BONE. BY WILLIAM STOKES, F.R.C.S.I.,

Professor of Surgery, Royal College of Surgeons of Ireland.

EARLY in October of last year, J. T., aged 47 , a farmer by occupation, was admitted into the Richmond Surgical Hospital, under my care, having been recommended to me by my friend Dr. G. Plunket O'Farrell. The patient was suffering from the existence of a large tumour intimately connected with the left superior maxilla. It extended chiefly into the mouth, filling to a great extent that cavity, resting on the tongue, and almost extending across to the right tonsil. This rendered both mastication and deglutition most difficult. Externally, there was, on the 'left side of the face, rather a fulness or tumefaction than any distinct tumour. Two points of apparent fluctuation on the surface of the tumour inside the mouth were observed, one to the left of the incisor teeth, and the other about the centre of the tumour. The patient first noticed the growth about eighteen months previously to his admission into Richmond Hospital; but, judging from the connections which I subsequently found, the tumour had probably existed for a much longer period. The patient did not suffer any pain; there were no ulcerations on its mucous surface, and, externally, the integuments presented a perfectly healthy appearance. His general health was unaffected. As the tumour was making a serious advance into the cavity of the mouth, to a great extent filling it up, I determined, strengthened by the coinciding views of my colleagues, to remove the growth; and, on October 30 th, the operation was performed. The incisors being extracted, an incision was carried from the inner angle of the eye along the side of the nose, then horizontally to the septum, and finally through the lip vertically downwards (Ferguson's incision). The flap was then dissected backwards. I then found, on making slight pressure on the anterior surface of the tumour, that the bone yielded, giving the sensation of parchment. I then dissected the nasal tissues towards the right side opening into the nasal fossa. The two superior maxillary bones were then separated 